\title{
Hybrid joint universality theorem for Dirichlet $L$-functions
}

\author{
by \\ Łukasz Pańkowski (Poznań)
}

1. Introduction. In 1975 Voronin [9] proved a remarkable universality theorem for the Riemann zeta function. This result was improved by Reich [6] and Bagchi [1] and can be formulated as follows: let $K$ be any compact subset of the strip $D:=\{s \in \mathbb{C}: 1 / 2<\Re(s)<1\}$ with connected complement and let $f(s)$ be a function non-vanishing and continuous on $K$ and analytic in the interior of $K$. Then, for every $\varepsilon>0$, the set of positive real numbers $\tau=\tau(\varepsilon)$ satisfying

$$
\max _{s \in K}|\zeta(s+i \tau)-f(s)|<\varepsilon
$$

has a positive lower density.

Recall that for a Lebesgue measurable set $A \subset(0, \infty)$ we define the natural density of $A$ as

$$
\lim _{T \rightarrow \infty} \frac{\mu(A \cap(0, T])}{T},
$$

if the limit exists, where $\mu$ denotes the Lebesgue measure on $\mathbb{R}$. Moreover, if the lower limit of the same quotient is positive, then we say that $A$ has a positive lower density.

Further research on universality theorems focused on replacing the Riemann zeta function by other $L$-functions of importance in analytic number theory. For details we refer to [5] and [7]. Let us mention only that Voronin in his Ph.D. thesis [8] proved a joint universality theorem for Dirichlet $L$-functions. Roughly speaking, it states that some imaginary shifts of Dirichlet $L$-functions associated with non-equivalent characters approximate simultaneously any functions which are non-vanishing and continuous on $K \subset\{s \in \mathbb{C}: 1 / 2<\sigma<1\}$ and holomorphic in the interior of $K$, where as before $K$ is a compact set with connected complement.

Known proofs of universality theorems depend on diophantine approximations, especially, on the Kronecker-Weyl theorem (proved in the qualita- 
tive and quantitative form by Kronecker and Weyl respectively) which could be stated as follows.

For $x \in \mathbb{R}$ let $\|x\|$ denote the distance from $x$ to the nearest integer. Then for arbitrary real numbers $\alpha_{1}, \ldots, \alpha_{n}$ linearly independent over $\mathbb{Q}$, any real numbers $\theta_{1}, \ldots, \theta_{n}$ and any positive numbers $\varepsilon_{1}, \ldots, \varepsilon_{n}$, the set of $\tau$ such that

$$
\left\|\tau \alpha_{i}-\theta_{i}\right\|<\varepsilon_{i} \quad \text { for all } 1 \leq i \leq n
$$

has a positive density, which is equal to $2^{n} \prod_{1 \leq i \leq n} \varepsilon_{i}$.

The following question arises in a natural way: does the set of real numbers $\tau$ satisfying simultaneously (1) and (2) have a positive lower density? A partial answer was given by Kaczorowski and Kulas [3] in 2007, who proved that if $K \subset\{s \in \mathbb{C}: 1 / 2<\sigma<1\}$ is a compact set with connected complement, $f_{1}, \ldots, f_{n}$ are any functions which are non-vanishing and continuous on $K$ and analytic in the interior, and $\chi_{1}, \ldots, \chi_{n}$ are pairwise non-equivalent Dirichlet characters, then, for every sequence $\left(\theta_{p}\right)_{p \leq z}$ of real numbers indexed by primes up to $z$ and for every $\varepsilon>0$, the set of positive real numbers $\tau$ satisfying the inequalities

$$
\max _{1 \leq j \leq n} \max _{s \in K}\left|L\left(s+i \tau, \chi_{j}\right)-f_{j}(s)\right|<\varepsilon, \quad \max _{p \leq z}\left\|\tau \frac{\log p}{2 \pi}-\theta_{p}\right\|<\varepsilon
$$

has a positive lower density.

Although this result is more general than the joint universality theorem, the second inequality is only a special case of the inequality in the KroneckerWeyl theorem. Therefore the purpose of this paper is to prove a theorem of the above type in full generality, with logarithms of primes replaced by an arbitrary set of real numbers linearly independent over $\mathbb{Q}$. More precisely, we prove the following theorem, which can be called the hybrid joint universality theorem.

Theorem 1.1. Let $K \subset\{s \in \mathbb{C}: 1 / 2<\sigma<1\}$ be any compact set with connected complement, $\chi_{1}, \ldots, \chi_{n}$ pairwise non-equivalent Dirichlet characters and $f_{1}, \ldots, f_{n}$ any functions which are non-vanishing and continuous on $K$ and analytic in the interior. Moreover, let $\left(\alpha_{i}\right)_{1 \leq i \leq m}$ be any sequence of real numbers linearly independent over $\mathbb{Q}$, and $\left(\theta_{i}\right)_{1 \leq i \leq m}$ any sequence of real numbers. Then, for every $\varepsilon>0$, the set of real numbers $\tau$ satisfying the inequalities

$$
\max _{1 \leq j \leq n} \max _{s \in K}\left|L\left(s+i \tau, \chi_{j}\right)-f_{j}(s)\right|<\varepsilon, \quad \max _{1 \leq i \leq m}\left\|\tau \alpha_{i}-\theta_{i}\right\|<\varepsilon
$$

has a positive lower density. 
In fact, we prove Theorem 1.1 in a more general form, with Dirichlet $L$-functions replaced by a finite family of general $L$-functions (called $a c$ ceptable) satisfying some natural analytic and arithmetic conditions like the Euler product representation, the Ramanujan conjecture and analytic continuation. For a precise definition of the family of acceptable functions see Section 2 below. The most general hybrid joint universality theorem is formulated as Theorem 4.2 .

We conclude the paper by presenting some consequences of our hybrid joint universality theorem. In particular, we show that no non-trivial density estimate for zeros of linear combinations of acceptable functions with general Dirichlet polynomials as coefficients can hold.

2. Preliminaries. As mentioned above, we prove our main theorem for some large class of functions, which contains the Dirichlet $L$-functions. We first define the class $\mathcal{E}$ (cf. [3, [4]) to consist of functions

$$
F(s)=\prod_{p} R_{p}\left(p^{-s}\right) \quad(\sigma>1),
$$

where $R_{p}(z)=1+\sum_{m=1}^{\infty} a\left(p^{m}\right) z^{m}$ are rational functions, analytic and nonvanishing on the disk $|z|<1$, which satisfy the following conditions:

(1) (Ramanujan conjecture) $\forall_{\varepsilon>0} a\left(p^{m}\right) \ll_{\varepsilon} p^{\varepsilon m}$ uniformly in $p$.

(2) $F$ has meromorphic continuation to the half-plane $\sigma>1 / 2$. It can have at most a finite number of poles and all of them lie on the straight line $\sigma=1$.

(3) $F$ is a function of finite order, which means that

$$
F(s) \ll_{\sigma}|t|^{A(\sigma)} \quad \text { for } \sigma>1 / 2,|t| \rightarrow \infty .
$$

(4) For any fixed $1 / 2<\sigma<1$ the square mean-value

$$
\frac{1}{T} \int_{-T}^{T}|F(\sigma+i t)|^{2} d t
$$

is bounded as $T \rightarrow \infty$.

Moreover, let $\Omega=\prod_{p} \mathbb{R}$ denote the set of all sequences $\Theta=\left(\theta_{p}\right)_{p}$ of real numbers indexed by primes.

For any finite set $M$ of prime numbers and any function $F \in \mathcal{E}$, we define for $\sigma>1 / 2$ the finite products

$$
F_{M}(s, \Theta)=\prod_{p \in M} R_{p}\left(p^{-s} e\left(-\theta_{p}\right)\right)
$$

and

$$
F(s)_{\mid M}=F(s) \prod_{p \in M} R_{p}^{-1}\left(p^{-s}\right) .
$$


Now, we are ready to define a class of finite sets of functions which is characterized by some kind of independence, and is of prime importance for us. Following [3] we call an open and bounded subset $G$ of $\mathbb{C}$ admissible when for every $\varepsilon>0$ the set $G_{\varepsilon}=\{s \in \mathbb{C}:|s-w|<\varepsilon$ for certain $w \in G\}$ has connected complement.

We say that a set $\left\{F_{1}, \ldots, F_{n}\right\} \subset \mathcal{E}$ is acceptable if it satisfies the following condition: For every finite set $M$ of prime numbers, every admissible domain $G$ such that $\bar{G} \subset\{s \in \mathbb{C}: 1 / 2<\Re(s)<1\}$, and any analytic and non-vanishing functions $f_{1}, \ldots, f_{n}$ on $\bar{G}$, there exists a sequence of finite sets $M_{1} \subset M_{2} \subset \cdots$ of primes such that

$$
\bigcup_{k=1}^{\infty} M_{k}=\{p: p \notin M\}
$$

and for certain $\Theta_{k} \in \Omega$, as $k \rightarrow \infty$,

$$
F_{j, M_{k}}\left(s, \Theta_{k}\right) \rightarrow f_{j}(s) \quad \text { uniformly for } s \in \bar{G}, j=1, \ldots, n .
$$

REMARK 2.1. As in the proof of [3, Lemma 7] one can prove that the Dirichlet $L$-functions for pairwise non-equivalent characters form an acceptable subset of $\mathcal{E}$. We skip the proof of this fact as the modifications needed are straightforward and can be left to the reader.

To end this section, let us recall some properties of the sequence $\frac{\log p}{2 \pi}$ where $p$ runs over primes, which play an important role in the proof of the hybrid joint universality theorem.

First of all, note that the numbers $\frac{\log p}{2 \pi}$ are linearly independent over $\mathbb{Q}$, so we can use the Kronecker-Weyl approximation theorem. The main consequence is that for every finite set $M$ of primes the curve

$$
\gamma(\tau)=\left(\frac{\tau \log p}{2 \pi}\right)_{p \in M}, \quad \tau>0,
$$

is uniformly distributed mod 1 in $\mathbb{R}^{\sharp M}$. Obviously, we can say the same about any sequence linearly independent over $\mathbb{Q}$.

Recall that a curve $\gamma:(0, \infty) \rightarrow \mathbb{R}^{n}$ is uniformly distributed $\bmod 1$ in $\mathbb{R}^{n}$ if for every $n$-dimensional interval $\Pi=\left[\alpha_{1}, \beta_{1}\right] \times \cdots \times\left[\alpha_{n}, \beta_{n}\right] \subset[0,1]^{n}$ we have

$$
\lim _{T \rightarrow \infty} \frac{1}{T} \mu\{\tau \in(0, T]: \gamma(\tau) \in \Pi \bmod 1\}=\prod_{j=1}^{n}\left(\beta_{j}-\alpha_{j}\right),
$$

where $\gamma(\tau) \in \Pi \bmod 1$ means that there exists $y \in \mathbb{Z}^{n}$ such that $\gamma(\tau)-y \in \Pi$.

The following lemma plays a crucial role in the proof of our main theorem.

LEMMA 2.2. Let $\left(\alpha_{i}\right)_{1 \leq i \leq m}$ be a sequence of real numbers linearly independent over $\mathbb{Q}$, and $\left(\theta_{i}\right)_{1 \leq i \leq m}$ any real numbers. Then there exist a finite set $B=B\left(\alpha_{1}, \ldots, \alpha_{m}\right)$ of primes and a sequence $\left(\theta_{p}^{*}\right)_{p \in B}$ of real numbers such 
that for every finite set $M$ of primes with $M \cap B=\emptyset$, any real numbers $\theta_{p}$, $p \in M$, and every $\varepsilon>0$, the set of real numbers $\tau$ satisfying the inequalities

$$
\begin{gathered}
\max _{1 \leq i \leq m}\left\|\tau \alpha_{i}-\theta_{i}\right\|<\varepsilon, \\
\max _{p \in M}\left\|\tau \frac{\log p}{2 \pi}-\theta_{p}\right\|<\varepsilon, \quad \max _{p \in B}\left\|\tau \frac{\log p}{2 \pi}-\theta_{p}^{*}\right\|<\varepsilon
\end{gathered}
$$

has a positive density, which can be explicitly calculated.

Proof. Let $\mathbb{P}$ denote the set of all primes and

$$
\mathbb{A}=\operatorname{span}_{\mathbb{Q}}\left\{\frac{\log p}{2 \pi}\right\}_{p \in \mathbb{P}}, \quad V=\operatorname{span}_{\mathbb{Q}}\left\{\alpha_{1}, \ldots, \alpha_{m}\right\},
$$

where $\operatorname{span}_{\mathbb{Q}}\left\{a_{i}\right\}$ denotes the vector space over $\mathbb{Q}$ generated by the real numbers $a_{i}$.

Obviously, if $V \cap \mathbb{A}=\{0\}$, then the elements $\frac{\log p}{2 \pi}, p \in \mathbb{P}$, and $\alpha_{i}, 1 \leq$ $i \leq m$, are linearly independent over $\mathbb{Q}$. Hence, putting $B=\emptyset$ and using the Kronecker-Weyl theorem yields the assertion.

Therefore, assume that the vector space $V \cap \mathbb{A}$ is non-trivial and has a basis $\left\{\beta_{1}, \ldots, \beta_{k}\right\}$. It is clear that we can complete $\left\{\beta_{1}, \ldots, \beta_{k}\right\}$ by elements $\alpha_{i}$ to a basis of $V$, say

$$
\left\{\beta_{1}, \ldots, \beta_{k}, \alpha_{k+1}, \ldots, \alpha_{m}\right\}
$$

Observe that

$$
\operatorname{span}_{\mathbb{Q}}\left\{\alpha_{k+1}, \ldots, \alpha_{m}\right\} \cap \mathbb{A}=\{0\} .
$$

It is also clear that $\{k+1, \ldots, m\}$ is the largest set of indices for which (3) holds.

Now, let $B=\left\{p_{1}, \ldots, p_{r}\right\}$ be the minimal set of primes such that

$$
\beta_{1}, \ldots, \beta_{k} \in \operatorname{span}_{\mathbb{Q}}\left\{\frac{\log p_{1}}{2 \pi}, \ldots, \frac{\log p_{r}}{2 \pi}\right\} .
$$

Notice that $k \leq r<\infty$ and

$$
\alpha_{1}, \ldots, \alpha_{k} \in V \subset \operatorname{span}_{\mathbb{Q}}\left\{\frac{\log p_{1}}{2 \pi}, \ldots, \frac{\log p_{r}}{2 \pi}, \alpha_{k+1}, \ldots, \alpha_{m}\right\} .
$$

Thus, there exists a positive integer $N$ such that for all $1 \leq i \leq k$ we have

$$
N \alpha_{i}=\sum_{j=1}^{r} n_{i j} \frac{\log p_{j}}{2 \pi}+\sum_{j=k+1}^{m} m_{i j} \alpha_{j}
$$

for suitable integers $n_{i j}$ and $m_{i j}$.

By the linear independence of $\alpha_{1}, \ldots, \alpha_{m}$ it is easy to see that the vectors $\left(n_{i 1}, n_{i 2}, \ldots, n_{i r}\right), i=1, \ldots, k$, are linearly independent over $\mathbb{Q}$. Hence, as 
$k \leq r$, for any real numbers $b_{1}, \ldots, b_{k}$ the matrices

$$
A=\left(n_{i j}\right)_{1 \leq i \leq k ; 1 \leq j \leq r}, \quad(A \mid b)=\left(n_{i j} \mid b_{i}\right)_{1 \leq i \leq k ; 1 \leq j \leq r},
$$

where $b^{T}=\left(b_{1}, \ldots, b_{k}\right)$, have rank $k$. Therefore for any real numbers $b_{1}, \ldots, b_{k}$ the system of linear equations

$$
A \cdot x=b
$$

has at least one solution.

Let $\theta_{p}^{\prime}, p \in B$, be a solution of the above system with

$$
b_{i}=\theta_{i}-\sum_{j=k+1}^{m} m_{i j} \frac{\theta_{j}}{N} \quad(i=1, \ldots, k) .
$$

In addition, we put $\theta_{p}^{*}=N \theta_{p}^{\prime}$ for $p \in B$.

Now, recalling (3), we see that the elements of the set

$$
\left\{N \frac{\log p}{2 \pi}\right\}_{p \in M} \cup\left\{\frac{\log p}{2 \pi}\right\}_{p \in B} \cup\left\{\alpha_{k+1}, \ldots, \alpha_{m}\right\}
$$

are linearly independent over $\mathbb{Q}$ for every finite set $M$ of primes with $M \cap B=\emptyset$.

Hence, by the Kronecker-Weyl theorem, for any real numbers $\theta_{p}, p \in M$, the set of real numbers $\tau$ satisfying the inequalities

$$
\begin{aligned}
& \max _{k+1 \leq i \leq m}\left\|\tau \alpha_{i}-\frac{\theta_{i}}{N}\right\|<\frac{\varepsilon}{N}, \\
& \max _{p \in M}\left\|N \tau \frac{\log p}{2 \pi}-\theta_{p}\right\|<\varepsilon, \\
& \max _{p \in B}\left\|\tau \frac{\log p}{2 \pi}-\theta_{p}^{\prime}\right\|<\frac{\varepsilon}{N}
\end{aligned}
$$

has a positive density, which can be explicitly calculated. Moreover, by the definition of $\theta_{p}^{\prime}, p \in B$, and the inequalities (4) and (6), we see that for those $\tau$,

$$
\max _{1 \leq i \leq k}\left\|N \tau \alpha_{i}-\theta_{i}\right\|<\varepsilon .
$$

Thus, multiplying (6) by $N$ completes the proof.

\section{The main lemma}

Lemma 3.1. Let $G$ be an admissible domain such that $\bar{G} \subset\{s \in \mathbb{C}$ : $1 / 2<\sigma<1\}$, and $\left\{F_{1}, \ldots, F_{n}\right\} \subset \mathcal{E}$ be any acceptable set. Moreover, let $\left(\alpha_{i}\right)_{1 \leq i \leq m}$ be real numbers linearly independent over $\mathbb{Q},\left(\theta_{i}\right)_{1 \leq i \leq m}$ any real numbers, and $f_{1}, \ldots, f_{n}$ functions which are analytic and non-vanishing 
on $\bar{G}$. Then, for every $\varepsilon>0$ and any set $G_{0} \subset \overline{G_{0}} \subset G$, there exist a finite set $B=B\left(\alpha_{1}, \ldots, \alpha_{m}\right)$ of primes and a sequence $\left(\theta_{p}^{*}\right)_{p \in B}$ of real numbers such that the set of positive real numbers $\tau$ satisfying the inequalities

$$
\begin{gathered}
\max _{1 \leq j \leq n} \max _{s \in \overline{G_{0}}}\left|F_{j}(s+i \tau)_{\mid B}-f_{j}(s) \prod_{p \in B} R_{p}^{-1}\left(p^{-s} e\left(-\theta_{p}^{*}\right)\right)\right|<\varepsilon, \\
\max _{p \in B}\left\|\tau \frac{\log p}{2 \pi}-\theta_{p}^{*}\right\|<\varepsilon, \quad \max _{1 \leq i \leq m}\left\|\tau \alpha_{i}-\theta_{i}\right\|<\varepsilon
\end{gathered}
$$

has a positive lower density.

Proof. We closely follow the proof of Lemma 1, Chapter 7 in [4, and hence we shall be very brief.

First, using Lemma 2.2 we choose a set $B=B\left(\alpha_{1}, \ldots, \alpha_{m}\right)$ and real numbers $\theta_{p}^{*}, p \in B$. Moreover, let $J \subset\{1, \ldots, m\}$ be a maximal set of indices such that the elements of the set

$$
\left\{\alpha_{i}\right\}_{i \in J} \cup\left\{\frac{\log p}{2 \pi}\right\}_{p \in \mathbb{P}}
$$

are linearly independent over $\mathbb{Q}$. Hence, as in the proof of Lemma 2.2 , it is clear that the elements $\alpha_{i}, i \in J$, complete a basis of $V \cap \operatorname{span}_{\mathbb{Q}}\left\{\frac{\log p}{2 \pi}\right\}_{p \in \mathbb{P}}$ to a basis of $V$.

Let

$$
\widetilde{f}_{j}(s)=f_{j}(s) \prod_{p \in B} R_{p}^{-1}\left(p^{-s} e\left(-\theta_{p}^{*}\right)\right)
$$

Fix $\varepsilon>0$. Then from the definition of acceptability we see that there exist a sequence $\Theta_{k}=\left(\theta_{p}^{(k)}\right) \in \Omega$ and a large integer $k_{0}$ such that for each $k \geq k_{0}$,

$$
\max _{1 \leq j \leq n} \max _{s \in \bar{G}}\left|F_{j, M_{k}}\left(s, \Theta_{k}\right)-\widetilde{f}_{j}(s)\right|<\varepsilon,
$$

where $M_{k}$ is a finite set of primes $p \notin B$.

Let $k$ denote a generic integer greater than or equal to $k_{0}$ and let $N$ have the same meaning as in the proof of Lemma 2.2. By continuity, if $\max _{p \in M_{k}}\left\|\tau \frac{N \log p}{2 \pi}-\theta_{p}^{(k)}\right\|<\delta$ for sufficiently small $\delta>0$, then

$$
\max _{1 \leq j \leq n} \max _{s \in \bar{G}}\left|F_{j, M_{k}}(s+i N \tau, 0)-\widetilde{f}_{j}(s)\right|<\varepsilon .
$$

Let $Q=\{p: p<y\} \backslash B$, where $y$ is so large that $M_{k} \subset Q$. Now, using the last lemma (see (4)-(6) and the fact that $M_{k} \cap B=\emptyset$ we see that the 
set $A$ of positive numbers $\tau$ satisfying the inequalities

$$
\begin{aligned}
\max _{i \in J}\left\|\tau \alpha_{i}-\frac{\theta_{i}}{N}\right\| & <\frac{\varepsilon}{N}, \\
\max _{p \in M_{k}}\left\|N \tau \frac{\log p}{2 \pi}-\theta_{p}^{(k)}\right\| & <\delta, \\
\max _{p \in B}\left\|\tau \frac{\log p}{2 \pi}-\frac{\theta_{p}^{*}}{N}\right\| & <\frac{\varepsilon}{N}
\end{aligned}
$$

has a positive density, which is equal to $\mu(\mathcal{D})$, where

$$
\begin{aligned}
\mathcal{D}=\left\{\bar{\omega}=\left(\omega_{i}^{(1)}\right)_{i \in J} \frown\right. & \left(\omega_{p}^{(2)}\right)_{p \in B \cup Q} \subset[0,1]^{|J|+y}: \max _{i \in J}\left\|\omega_{i}^{(1)}-\frac{\theta_{i}}{N}\right\|<\frac{\varepsilon}{N}, \\
& \left.\max _{p \in M_{k}}\left\|\omega_{p}^{(2)}-\theta_{p}^{(k)}\right\|<\delta, \max _{p \in B}\left\|\omega_{p}^{(2)}-\frac{\theta_{p}^{*}}{N}\right\|<\frac{\varepsilon}{N}\right\} .
\end{aligned}
$$

Here $\frown$ denotes concatenation of sequences and $\mu$ denotes the $(\sharp J+y)$ dimensional Lebesgue measure.

Consider now $I=\sum_{j=1}^{n} I_{j}$, where

$$
I_{j}=\frac{1}{T} \int_{A_{T}}\left(\iint_{G}\left|F_{j}(s+i N \tau)_{\mid B}-F_{j, M_{k}}(s+i N \tau, 0)\right|^{2} d b\right) d \tau,
$$

with $d b=d \sigma d t$ and $A_{T}=A \cap\left[T_{0}, T\right]$. We choose $T_{0}$ so large that $F_{j}$ are analytic on $\sigma>1 / 2,|t|>T_{0}$.

Obviously

$$
I \leq 2 S_{1}+2 S_{2},
$$

where

$$
\begin{aligned}
& S_{1}=\frac{1}{T} \int_{A_{T}}\left(\iint_{G} \sum_{j=1}^{n}\left|F_{j, Q}(s+i N \tau, 0)-F_{j, M_{k}}(s+i N \tau, 0)\right|^{2} d b\right) d \tau, \\
& S_{2}=\frac{1}{T} \int_{A_{T}}\left(\iint_{G} \sum_{j=1}^{n}\left|F_{j}(s+i N \tau)_{\mid B}-F_{j, Q}(s+i N \tau, 0)\right|^{2} d b\right) d \tau .
\end{aligned}
$$

We intend to show that $I \ll \varepsilon^{2}$.

First we handle $S_{1}$, which can be written as

$$
S_{1}=\sum_{j=1}^{n} \iint_{G}\left(\frac{1}{T} \int_{A_{T}}\left|F_{j, Q}\left(s,\left(\tau \frac{N \log p}{2 \pi}\right)\right)-F_{j, M_{k}}\left(s,\left(\tau \frac{N \log p}{2 \pi}\right)\right)\right|^{2} d \tau\right) d b .
$$

By the Kronecker-Weyl theorem and the definition of the set $J$, the curve

$$
\gamma(\tau)=\left(\tau \alpha_{i}\right)_{i \in J} \frown\left(\tau \frac{\log p}{2 \pi}\right)_{p \in B} \frown\left(\tau \frac{N \log p}{2 \pi}\right)_{p \in Q}
$$

is uniformly distributed mod 1. 
Then, by Theorem A.8.3 in [4], we have

$$
\begin{aligned}
& \lim _{T \rightarrow \infty} \frac{1}{T} \int_{A_{T}}\left|F_{j, Q}\left(s,\left(\tau \frac{N \log p}{2 \pi}\right)\right)-F_{j, M_{k}}\left(s,\left(\tau \frac{N \log p}{2 \pi}\right)\right)\right|^{2} d \tau \\
& \leq\left(\max _{s \in \bar{G}}\left|\widetilde{f}_{j}(s)\right|+2 \varepsilon\right)^{2} \int_{\mathcal{D}} \ldots \int_{j, Q \backslash M_{k}}(s, \bar{\omega})-\left.1\right|^{2} d \mu,
\end{aligned}
$$

where $\mu$ denotes the Lebesgue measure on $\mathbb{R}^{|J|+y}$.

Observe now that from the definition of $\mathcal{D}$ we have

$$
\int_{\mathcal{D}}^{\ldots}\left|F_{j, Q \backslash M_{k}}(s, \bar{\omega})-1\right|^{2} d \mu=\mu(\mathcal{D}) \int_{0}^{1} \cdots \int_{0}^{1}\left|F_{j, Q \backslash M_{k}}(s, \bar{\omega})-1\right|^{2} d \mu .
$$

Finally, if we write $F_{j, Q \backslash M_{k}}(s, \bar{\omega})-1$ as a Dirichlet series and assume that $M_{k}$ contains all primes less than $y_{k}$, then after some easy calculations we obtain

$$
\int_{0}^{1} \cdots \int_{0}^{1}\left|F_{j, Q \backslash M_{k}}(s, \bar{\omega})-1\right|^{2} d \mu \leq \sum_{m>y_{k}} \frac{1}{m^{1+\varepsilon_{1}}},
$$

where $\varepsilon_{1}<\frac{2}{3} \operatorname{dist}(\bar{G}, 1 / 2)$. Hence for sufficiently large $T$ and $k$ we have

$$
S_{1}<\frac{1}{4} \mu(\mathcal{D}) \varepsilon^{2} .
$$

Arguing analogously to [4] we prove a similar estimate to $S_{2}$. The modifications needed are easy and can be left to the reader. As a result we obtain

$$
I \leq \mu(\mathcal{D}) \varepsilon^{2} \quad \text { as } T \rightarrow \infty .
$$

On the other hand, we know that

$$
\lim \frac{1}{T} \int_{A_{T}} d \tau=\mu(\mathcal{D})
$$

Finally, by Theorem A.2.7 from [4], there exists a set $Y \subset A_{T}$ such that $\mu(Y) \gg T$ and for all $\tau \in Y$,

$$
\max _{1 \leq j \leq n} \max _{s \in \overline{G_{0}}}\left|F_{j}(s+i N \tau)_{\mid B}-F_{j, M_{k}}(s+i N \tau, 0)\right|^{2}<2 \varepsilon^{2} .
$$

Hence, recalling (7), (8), and multiplying (9) by $N$ completes the proof.

4. The main theorem. In the proof of Theorem 4.2, which we regard as the main result of this paper, we make use of the following famous Mergelyan theorem [2].

LemmA 4.1. Let $K \subset \mathbb{C}$ be a compact set with connected complement and $f: K \rightarrow \mathbb{C}$ any function continuous on $K$ and analytic in the interior 
of $K$. Then, for every $\varepsilon>0$, there exists a polynomial $P$ such that

$$
\max _{s \in K}|f(s)-P(s)|<\varepsilon .
$$

Theorem 1.1 is an immediate consequence of the following result and Remark 2.1

Theorem 4.2 (hybrid joint universality theorem). Let $K \subset\{s \in \mathbb{C}$ : $1 / 2<\sigma<1\}$ be a compact set with connected complement, $\left\{F_{1}, \ldots, F_{n}\right\} \subset \mathcal{E}$ an acceptable set and $f_{1}, \ldots, f_{n}$ any functions continuous and non-vanishing on $K$ which are analytic in the interior of $K$. Moreover, let $\left(\alpha_{i}\right)_{1 \leq i \leq m}$ be any fixed sequence of real numbers linearly independent over $\mathbb{Q}$. Then, for every $\varepsilon>0$ and any real numbers $\left(\theta_{i}\right)_{1 \leq i \leq m}$, there exists a set $A$ with a positive lower density such that for all $\tau \in A$,

$$
\begin{gathered}
\max _{1 \leq j \leq n} \max _{s \in K}\left|F_{j}(s+i \tau)-f_{j}(s)\right|<\varepsilon, \\
\max _{1 \leq i \leq m}\left\|\tau \alpha_{i}-\theta_{i}\right\|<\varepsilon .
\end{gathered}
$$

Proof. As in the proof of Theorem 3 in [3], by the Mergelyan theorem, we can assume that $K=\bar{G}$ for some admissible set $G$ and $f_{j}$ are analytic and non-vanishing on some simply connected set $G_{1}$ such that $\bar{G} \subset G_{1} \subset$ $\overline{G_{1}} \subset\{s: 1 / 2<\sigma<1\}$.

Let $B$ have the same meaning as in Lemma 3.1 and let $n_{1}$ denote the number of its elements. Moreover, let

$$
\begin{gathered}
m_{0}=\max _{1 \leq j \leq N} \max _{s \in \overline{G_{1}}}\left|f_{j}(s)\right|, \quad m_{1}=1+n_{1} C^{n_{1}-1} m_{0}, \\
P=\prod_{p \in B}\left(1+\sum_{m=1}^{\infty}\left|a\left(p^{m}\right)\right| p^{-1 / 2}\right),
\end{gathered}
$$

where

$$
0<C^{-1}<\min _{p \in B} \min _{|z| \leq 1 / \sqrt{2}}\left|R_{p}(z)\right| .
$$

By continuity, for any $\varepsilon>0$, we find $\delta=\delta(\varepsilon)$ such that

$$
\max _{p \in B} \max _{s \in \bar{G}}\left|R_{p}^{-1}\left(p^{-s} e\left(-\Theta_{1}\right)\right)-R_{p}^{-1}\left(p^{-s} e\left(-\Theta_{2}\right)\right)\right|<\frac{\varepsilon}{m_{1} P},
$$

whenever $\left\|\theta_{p}^{(1)}-\theta_{p}^{(2)}\right\| \leq \delta$ for $p \in B$.

Now, using Lemma 3.1, we see that the set of $\tau$ satisfying

$$
\max _{1 \leq j \leq n} \max _{s \in \bar{G}}\left|F_{j}(s+i \tau)_{\mid B}-f_{j}(s) \prod_{p \in B} R_{p}^{-1}\left(p^{-s} e\left(-\theta_{p}^{*}\right)\right)\right|<\frac{\varepsilon}{m_{1} P},
$$




$$
\begin{gathered}
\max _{1 \leq i \leq m}\left\|\tau \alpha_{i}-\theta_{i}\right\|<\varepsilon, \\
\max _{p \in B}\left\|\tau \frac{\log p}{2 \pi}-\theta_{p}^{*}\right\|<\delta
\end{gathered}
$$

has a positive lower density.

Using (13) and the inequality

$$
\left|\prod_{j=1}^{n} a_{j}-\prod_{j=1}^{n} b_{j}\right| \leq R^{n-1} \sum_{j=1}^{n}\left|a_{j}-b_{j}\right|
$$

which holds for any complex numbers $a_{j}, b_{j}, j=1, \ldots, n$, such that $\left|a_{j}\right|,\left|b_{k}\right|$ $\leq R$, we deduce for $s \in \bar{G}$ that

$$
\begin{aligned}
\mid f_{j}(s) \prod_{p \in B} R_{p}^{-1}\left(p^{-s}\right. & \left.e\left(-\theta_{p}^{*}\right)\right)-f_{j}(s) \prod_{p \in B} R_{p}^{-1}\left(p^{-s-i \tau}\right) \mid \\
& \leq m_{0}\left|\prod_{p \in B} R_{p}^{-1}\left(p^{-s} e\left(-\theta_{p}^{*}\right)\right)-\prod_{p \in B} R_{p}^{-1}\left(p^{-s-i \tau}\right)\right| \\
& \leq m_{0} n_{1} C^{n_{1}-1} \max _{p \in B}\left|R_{p}^{-1}\left(p^{-s} e\left(-\theta_{p}^{*}\right)\right)-R_{p}^{-1}\left(p^{-s-i \tau}\right)\right| \\
& \leq\left(1-\frac{1}{m_{1}}\right) \frac{\varepsilon}{P} .
\end{aligned}
$$

Consequently, using (11) we have

$$
\max _{1 \leq j \leq n} \max _{s \in \bar{G}}\left|F_{j}(s+i \tau)_{\mid B}-f_{j}(s) \prod_{p \in B} R_{p}^{-1}\left(p^{-s-i \tau}\right)\right|<\frac{\varepsilon}{P} .
$$

Multiplying the last inequality by $\left|\prod_{p \in B} R_{p}\left(p^{-s-i \tau}\right)\right|$ and noticing that this factor is $\leq P$, we obtain 10 , and the result follows.

5. Some applications. We first briefly present two evident consequences of the main theorem. We omit details of the proofs because they are almost the same as in [4, Chapter VII, Section 3.4].

Corollary 5.1. Let $\left\{F_{1}, \ldots, F_{n}\right\} \in \mathcal{E}$ be an acceptable set, and $\alpha_{1}, \ldots, \alpha_{m}$ real numbers linearly independent over $\mathbb{Q}$. Then the image of the function $\gamma: \mathbb{R} \rightarrow \mathbb{C}^{n k} \times \mathbb{T}^{m}$ given by

$$
\gamma(\tau)=\left(F_{1}(\sigma+i \tau), \ldots, F_{n}^{(k-1)}(\sigma+i \tau), e^{\alpha_{1} i \tau}, \ldots, e^{\alpha_{m} i \tau}\right)
$$

is everywhere dense in $\mathbb{C}^{n k} \times \mathbb{T}^{m}$, where $\mathbb{T}=\{s \in \mathbb{C}:|s|=1\}$.

Corollary 5.2. Let $F_{1}, \ldots, F_{n}, \alpha_{1}, \ldots, \alpha_{m}$ and $\mathbb{T}$ be as in Corollary 5.1. Suppose $G_{j}(1 \leq j \leq N)$ are continuous functions on $\mathbb{C}^{n k} \times \mathbb{T}^{m}$ such that

$$
\sum_{j=1}^{N} s^{j-1} G_{j}\left(F_{1}(\sigma+i \tau), \ldots, F_{n}^{(k-1)}(\sigma+i \tau), e^{\alpha_{1} i \tau}, \ldots, e^{\alpha_{m} i \tau}\right)=0
$$

for all $s \in \mathbb{C}$. Then the functions $G_{j}, j=1, \ldots, N$, vanish identically. 
Proof. Proceeding by contradiction it suffices to choose an open set $U \subset$ $\mathbb{C}^{n k} \times \mathbb{T}^{m}$ consisting of vectors $z$ for which

$$
\left|G_{j}(z)\right|>\delta>0 \text { for some } 1 \leq j \leq N .
$$

Then, by Corollary 5.1, there exists a complex number $s=\sigma+i \tau$ such that

$$
\left(F_{1}(\sigma+i \tau), \ldots, F_{n}^{(k-1)}(\sigma+i \tau), e^{\alpha_{1} i \tau}, \ldots, e^{\alpha_{m} i \tau}\right) \in U,
$$

and the result follows.

Finally, we generalize Theorem 2 from [3]. By a general Dirichlet polynomial we understand a function of the form

$$
P(s)=\sum_{i=1}^{n} a_{i} e^{-\lambda_{i} s}, \quad \text { where } a_{i} \in \mathbb{C}, \lambda_{i} \in \mathbb{R} .
$$

Corollary 5.3. Let $\left\{F_{1}, \ldots, F_{n}\right\} \subset \mathcal{E}$ be an acceptable set and let $P_{1}, \ldots, P_{n}$ be non-zero general Dirichlet polynomials. Then for $n \geq 2$ the function

$$
F(s)=\sum_{j=1}^{n} P_{j}(s) F_{j}(s)
$$

has infinitely many zeros off the critical line $\sigma=1 / 2$. Moreover, for every $1 / 2<\sigma_{1}<\sigma_{2}<1$ the number of zeros of $F(s)$ in the rectangle $\sigma_{1} \leq \sigma \leq \sigma_{2}$, $0<t<T$ is $\gg T$ as $T \rightarrow \infty$.

Proof. Assume that

$$
P_{j}(s)=\sum_{l=1}^{k_{j}} a_{l}^{(j)} e^{-\lambda_{l}^{(j)} s} .
$$

Let $\left\{\alpha_{i}\right\}_{1 \leq i \leq m}$ be a basis of the vector space over $\mathbb{Q}$ generated by all $\lambda_{l}^{(j)}$, $1 \leq j \leq n, 1 \leq l \leq k_{j}$. Choose an integer $N$ such that for each $1 \leq j \leq n$ and $1 \leq l \leq k_{j}$,

$$
\lambda_{l}^{(j)}=\sum_{1 \leq i \leq m} n_{i}^{(l, j)} \frac{\alpha_{i}}{N}, \quad n_{i}^{(l, j)} \in \mathbb{Z} .
$$

We now follow closely the proof of Theorem 2 in [3]. Let $\sigma_{0}$ be such that $1 / 2<\sigma_{0}<1$. It is sufficient to show that for every $\delta_{0}>0$ the function $F(s)$ has $\gg T$ zeros in $\left|\sigma-\sigma_{0}\right|<\delta_{0},|t| \leq T$.

Since $\prod_{j=1}^{n} P_{j}(s)$ does not vanish identically, there exist $t_{0}>0$ and $0<$ $\delta<1 / 2$ such that $P_{j}(s) \neq 0$ in the disk $\left|s-s_{0}\right| \leq \delta$, where $s_{0}=\sigma_{0}+i t_{0}$. Let $m_{0}=\min _{1 \leq j \leq n} \min _{\left|s-s_{0}\right| \leq \delta}\left|P_{j}(s)\right|$, and let $A_{j}$ be such that

$$
\sum_{j=0}^{n-1} A_{j} s^{j}=\left(s-s_{0}\right)^{n-1} \text {. }
$$


We define some constants:

$$
\begin{aligned}
& H_{0}=\max _{j}\left|A_{j}\right|, \quad m_{1}=\max _{j} \max _{1 / 2 \leq \sigma \leq 1} \sum_{i=1}^{k_{j}}\left|a_{i}^{(j)}\right| e^{-\lambda_{i}^{(j)} \sigma}, \quad m_{2}=2 \pi \max _{i, j, l} n_{i}^{(l, j)}, \\
& V=\left(2+\left|t_{0}\right|\right)^{n}, \quad M_{1}=\max \left(\frac{2 m_{1} m_{2}}{m_{0}}, N\left(m_{1}+\frac{2 H_{0} V m_{1}^{2} m_{2}}{m_{0}^{2}}\right)\right) .
\end{aligned}
$$

Using Theorem 4.2 we obtain a set of real numbers $\tau$ satisfying

$$
\begin{gathered}
\max _{1 \leq j \leq n} \max _{\left|s-s_{0}\right| \leq \delta}\left|F_{j}(s+i \tau)-\frac{A_{j-1} s^{j-1}}{P_{j}(s)}\right| \leq \frac{\delta^{n-1}}{M_{1}}, \\
\max _{1 \leq i \leq m}\left\|\frac{\tau \alpha_{i}}{2 N \pi}\right\| \leq \frac{\delta^{n-1}}{M_{1}} .
\end{gathered}
$$

For these $\tau$ and for $\left|s-s_{0}\right| \leq \delta$ we have

$$
\left|P_{j}(s+i \tau)-P_{j}(s)\right| \leq \max _{1 / 2 \leq \sigma \leq 1} \sum_{i=1}^{k_{j}}\left|a_{i}^{(j)}\right| \cdot e^{-\lambda_{i}^{(j)} \sigma} \cdot\left|e^{-i \tau \lambda_{i}^{(j)}}-1\right| .
$$

From the choice of $N$ and inequality (14) for $R=1$ we have

$$
\left|e^{-i \tau \lambda_{i}^{(j)}}-1\right| \leq \frac{m_{2} \delta^{n-1}}{M_{1}},
$$

hence

$$
\left|P_{j}(s+i \tau)-P_{j}(s)\right| \leq \frac{m_{1} m_{2} \delta^{n-1}}{M_{1}} .
$$

Further, omitting some technical details (cf. [3]), we obtain

$$
\left|P_{j}(s+i \tau) F_{j}(s+i \tau)-A_{j-1} s^{j-1}\right|<\frac{\delta^{n-1}}{n} .
$$

Finally, we see that the set of real numbers $\tau$ satisfying

$$
\max _{\left|s-s_{0}\right|=\delta}\left|F(s+i \tau)-\left(s-s_{0}\right)^{n-1}\right|<\delta^{n-1}
$$

has a positive lower density, and the result follows from the classical Rouché theorem.

Acknowledgments. The author would like to cordially thank Professor Jerzy Kaczorowski for helpful discussions and suggestions.

This research was partially supported by the grant no. N N201 148233 from the Polish Ministry of Science and Higher Education.

\section{References}

[1] B. Bagchi, The statistical behaviour and universality properties of the Riemann zetafunction and other allied Dirichlet series, Ph.D. Thesis, Indian Statistical Institute, Calcutta, 1981. 
[2] D. Gaier, Vorlesungen über Approximation im Komplexen, Birkhäuser, Basel, 1980.

[3] J. Kaczorowski and M. Kulas, On the non-trivial zeros off the critical line for Lfunctions from the extended Selberg class, Monatsh. Math. 150 (2007), 217-232.

[4] A. A. Karatsuba and S. M. Voronin, The Riemann Zeta Function, de Gruyter, Berlin, 1992.

[5] A. Laurinčikas, The universality of zeta-functions, Acta Appl. Math. 78 (2003), 251271.

[6] A. Reich, Universelle Wertverteilung von Eulerprodukten, Nachr. Akad. Wiss. Göttingen Math.-Phys. Kl. 1977, no. 1, 1-17.

[7] J. Steuding, Value-Distribution of L-functions, Springer, Berlin, 2007.

[8] S. M. Voronin, Analytic properties of Dirichlet generating functions of arithmetic objects, Ph.D. Thesis, Steklov Math. Inst., Moscow, 1977 (in Russian).

[9] - , Theorem on the universality of the Riemann zeta function, Izv. Akad. Nauk SSSR Ser. Mat. 39 (1975), 475-486 (in Russian); English transl.: Math. USSR-Izv. 9 (1975), 443-453.

Łukasz Pańkowski

Faculty of Mathematics and Computer Science

Adam Mickiewicz University

Umultowska 87

61-614 Poznań, Poland

E-mail: lpan@amu.edu.pl

Received on 15.10.2008

and in revised form on 17.6.2009 